\title{
ON A FORMULA OF BLOCH
}

William Duke \& ÖZlem Imamo $\overline{\mathrm{G}} \mathrm{LU}$

To Jean-Marc Deshouillers, on his sixtieth birthday

Abstract: We give a new proof of a formula of Bloch for a special value of a certain Eisenstein series of weight one with an additive character.

Keywords: Kronecker limit formulas, elliptic curves, dilogarithm.

\section{Introduction}

In his influential Irvine lecture notes of 1978, Bloch [2] expressed the value at $s=2$ of the $L$-function for a CM elliptic curve $E$ defined over $\mathbb{Q}$ in terms of a regulator map $K_{2}\left(E_{\mathbb{C}}\right) \rightarrow \mathbb{C}$. An important component of his argument is an analytic formula for a special value of an Eisenstein series of weight one with an additive character. Later Zagier [8] proved a generalization of this formula to other weights by computing the Fourier expansion of the Eisenstein series with respect to the additive character.

In this note we will deduce Bloch's formula from another Fourier expansion of an Eisenstein series, but in the $\tau$-variable and that holds for any value of $s$. Here $\tau \in \mathcal{H}$, the upper half-plane, determines the elliptic curve $E$ in the usual way. Our proof proceeds along lines similar to that given by Chowla and Selberg [3] of Kronecker's first limit formula. The Fourier expansion is given as Theorem 1 in $\S 3$ below. Although the technique we use to prove it is well-known, it seemed worthwhile to record this result, which does not appear to have been explicitly noted before. We will also clarify the relationship between Bloch's result and the Kronecker limit formulas.

2000 Mathematics Subject Classification: 11G55, 14G10.

Research of the first author supported in part by NSF Grant DMS-0355564. He thanks the Forschungsinstitut für Mathematik of ETH Zürich for its generous support and hospitality. 


\section{Bloch's formula}

After Zagier, Bloch's formula can be written for $u=\left(u_{1}, u_{2}\right) \in \mathbb{R}^{2}$ and $\tau=$ $x+i y \in \mathcal{H}$ as

$$
\frac{y^{2}}{\pi} \sum_{m, n \in \mathbb{Z}}^{\prime} \frac{e^{2 \pi i\left(n u_{2}-m u_{1}\right)}(m \tau+n)}{|m \tau+n|^{4}}=D(q ; z)+i J(q ; z),
$$

where the prime in the sum indicates that the term $(0,0)$ is to be omitted ${ }^{1}$. Here $q=\mathrm{e}(\tau)=e^{2 \pi i \tau}, z=\mathrm{e}\left(u_{1}+u_{2} \tau\right)$ and

$$
D(q ; z)=\sum_{\ell \in \mathbb{Z}} D\left(q^{\ell} z\right)
$$

where $D(z)$ is the Bloch-Wigner dilogarithm defined by

$$
D(z)=\arg (1-z) \log |z|-\operatorname{Im} \int_{0}^{z} \log (1-t) \frac{d t}{t} .
$$

Also,

$$
J(q ; z)=\sum_{\ell=0}^{\infty} J\left(q^{\ell} z\right)-\sum_{\ell=1}^{\infty} J\left(q^{\ell} z^{-1}\right)+\frac{\log ^{3}|z|}{3 \log |q|}-\frac{\log ^{2}|z|}{2}+\frac{\log |z| \log |q|}{6},
$$

where $J(z)=\log |z| \log |1-z|$.

Bloch's formula (1) should be compared with the Kronecker limit formulas ${ }^{2}$. Kronecker's first limit formula states that

$$
\lim _{s \rightarrow 1}\left(\frac{y^{s}}{2 \pi} \sum_{m, n \in \mathbb{Z}}^{\prime} \frac{1}{|m \tau+n|^{2 s}}-\frac{1}{2 s-2}\right)=\gamma-\log 2-\log \left(\sqrt{y}|\eta(\tau)|^{2}\right),
$$

where $\gamma$ is Euler's constant and $\eta(\tau)=q^{1 / 24} \prod_{n=1}^{\infty}\left(1-q^{n}\right)$ is the Dedekind eta function. This follows readily from the Fourier expansion

$$
\begin{aligned}
\pi^{-s} \Gamma(s) \frac{y^{s}}{2} \sum_{m, n}^{\prime}|m \tau+n|^{-2 s}= & y^{s} \varphi(s)+y^{1-s} \varphi(1-s) \\
& +2 \sqrt{y} \sum_{n, m \geqslant 1}\left(\frac{m}{n}\right)^{s-\frac{1}{2}} K_{s-\frac{1}{2}}(2 \pi m n y) \cos (2 \pi m n x),
\end{aligned}
$$

where $\varphi(s)=\pi^{-s} \Gamma(s) \zeta(2 s)$ and $K$ is the usual Bessel function, proved using the Poisson summation formula in one variable. To get the limit formula from this one uses the special value $[4$, p.978]

$$
K_{\frac{1}{2}}(t)=\sqrt{\frac{\pi}{2 t}} e^{-t}
$$

\footnotetext{
${ }^{1}$ We have corrected a sign error in the formula of Theorem 1 of [8].

${ }^{2}$ The best reference for the Kronecker limit formulas is [7]. See also [9, p.73].
} 
and the Taylor series $-\log (1-t)=\sum_{n \geqslant 1} t^{n} / n$, together with the well-known expansion of $\zeta(s)$ around $s=1$. A similar proof can be given of Kronecker's second limit formula, which states that for $u \notin \mathbb{Z}^{2}$

$$
\lim _{s \rightarrow 1} \frac{y^{s}}{2 \pi} \sum_{m, n \in \mathbb{Z}}^{\prime} \frac{e^{2 \pi i\left(n u_{2}-m u_{1}\right)}}{|m \tau+n|^{2 s}}=-\log |P(\tau, u)|,
$$

where again for $z=e\left(u_{1}+u_{2} \tau\right)$

$$
P(\tau, u)=q^{\frac{B_{2}\left(u_{2}\right)}{2}}(1-z) \prod_{n=1}^{\infty}\left(1-q^{n} z\right)\left(1-q^{n} z^{-1}\right) .
$$

Here $B_{n}(x)$ is the (periodic) Bernoulli polynomial. In the following we will show that Bloch's formula (1) can also be deduced from the Fourier series of an Eisenstein series.

Before proceeding to this, we remark that the imaginary part of Bloch's formula (1) can be written in a form more reminiscent of the second limit formula (6), namely:

$$
\frac{i y}{2 \pi^{2}} \sum_{m, n \in \mathbb{Z}}^{\prime} \frac{e^{2 \pi i\left(n u_{2}-m u_{1}\right)}(m x+n)}{|m \tau+n|^{4}}=\log |Q(\tau, u)|,
$$

where

$$
Q(\tau, u)=q^{\frac{B_{3}\left(u_{2}\right)}{3}}(1-z)^{u_{2}} \prod_{n=1}^{\infty} \frac{\left(1-q^{n} z\right)^{n+u_{2}}}{\left(1-q^{n} z^{-1}\right)^{n-u_{2}}} .
$$

Furthermore, by using the transformation $\tau \mapsto-1 / \tau$, we can express the elliptic dilogarithm (2) in terms of the infinite product $Q$ :

$$
\frac{1}{2 \pi} D(q ; z)=\log \left|Q\left(-1 / \tau, u^{\prime}\right)\right|-x \log |Q(\tau, u)|,
$$

where $u^{\prime}=\left(u_{2},-u_{1}\right)$. This is particularly simple in case $x=0$.

It is well known that $\log P(\tau, u)$ is the integral of a weight two holomorphic Eisenstein series and that its transformation properties under the modular group can be found by computing periods. Similarly, the transformation properties of $\log Q$ can be determined from those of an integral of a weight three holomorphic Eisenstein series; unfortunately they are complicated since we must perform only one integration, whereas it is the two-fold integral that transforms simply by a linear period polynomial. An interesting special case of the transformation under inversion appears in Ramaunujan's lost notebook (see [1]). 


\section{The Fourier expansion}

We now calculate the Fourier expansion of the Eisenstein series defined for $u, v \in \mathbb{R}^{2}$ and $\operatorname{Re}(s)>1$ by

$$
E_{u, v}(s, \tau)=y^{s} \sum_{\substack{m-v_{2} \in \mathbb{Z} \\ n-v_{1} \in \mathbb{Z}}}^{\prime} \frac{\mathrm{e}\left(n u_{2}-m u_{1}\right)(m \tau+n)}{|m \tau+n|^{2 s+1}} .
$$

Now $E_{u, v}^{*}(s, \tau)=\pi^{-s-1 / 2} \Gamma(s+1 / 2) E_{u, v}(s, \tau)$ is entire and satisfies the nice functional equation $[7, \mathrm{p} .71]$ :

$$
E_{u, v}^{*}(s, \tau)=e\left(v_{1} u_{2}-v_{2} u_{1}\right) E_{v, u}^{*}(1-s, \tau)
$$

We need the 1-dimensional version given for $\operatorname{Re} s>1$ and $a, b \in \mathbb{R}$ by

$$
\psi(s ; a, b)=\pi^{-s-\frac{1}{2}} \Gamma\left(s+\frac{1}{2}\right) \sum_{\substack{n-b \in \mathbb{Z} \\ n \neq 0}} \mathrm{e}(a n) n|n|^{-2 s-1},
$$

also entire with functional equation [7, p.69]

$$
\psi\left(\frac{1}{2}-s ; b, a\right)=i \mathrm{e}(a b) \psi(s ;-a, b)
$$

We will show in the next section that Bloch's formula (1) is an easy consequences of the following Fourier expansion. Let $(x)=0$ unless $x \in \mathbb{Z}$, when $(x)=1$.

Theorem 1. For $u, v \in \mathbb{R}^{2}, \tau \in \mathcal{H}$ and any $s \in \mathbb{C}$ we have

$$
E_{u, v}^{*}(s, \tau)=F_{u, v}(s, \tau)+e\left(v_{1} u_{2}-v_{2} u_{1}\right) F_{v, u}(1-s, \tau)
$$

where

$$
\begin{aligned}
F_{u, v}(s, \tau)= & \delta\left(v_{2}\right) y^{s} \psi\left(s ; u_{2}, v_{1}\right) \\
& -2 i y \mathrm{e}\left(v_{1} u_{2}\right) \sum_{\substack{m-v_{2} \in \mathbb{Z} \\
n+u_{2} \in \mathbb{Z} \\
m, n \neq 0}} \mathrm{e}\left(-m u_{1}+n v_{1}\right) n\left|\frac{m}{n}\right|^{1-s} K_{s-1}(2 \pi|m n| y) \mathrm{e}(m n x) .
\end{aligned}
$$

Proof. Write $E_{u, v}^{*}(s, \tau)=A+B$, where

$$
\begin{aligned}
& A=\pi^{-s-\frac{1}{2}} \Gamma\left(s+\frac{1}{2}\right) y^{s} \sum_{\substack{m-v_{2} \in \mathbb{Z} \\
n-v_{1} \in \mathbb{Z}}}^{\prime} \frac{\mathrm{e}\left(n u_{2}-m u_{1}\right)(m x+n)}{|m \tau+n|^{2 s+1}} \text { and } \\
& B=i \pi^{-s-\frac{1}{2}} \Gamma\left(s+\frac{1}{2}\right) y^{s+1} \sum_{\substack{m-v_{2} \in \mathbb{Z} \\
n-v_{1} \in \mathbb{Z}}}^{\prime} \frac{\mathrm{e}\left(n u_{2}-m u_{1}\right) m}{|m \tau+n|^{2 s+1}}
\end{aligned}
$$


Theorem 1 follows from the equations

$$
A=F_{u, v}(s, \tau) \quad \text { and } \quad B=e\left(v_{1} u_{2}-v_{2} u_{1}\right) F_{v, u}(1-s, \tau) .
$$

We will establish (17) by applying Poisson summation in both $A$ and $B$, but in a different way in each. From (15) we get that $A=A_{1}+A_{2}$ where $A_{1}=$ $\left(v_{2}\right) y^{s} \psi\left(s ; u_{2}, v_{1}\right)$ and

$$
\begin{aligned}
A_{2}= & y^{s} \sum_{\substack{m-v_{2} \in \mathbb{Z} \\
m \neq 0}} \mathrm{e}\left(-m u_{1}\right) \\
& \times \int_{0}^{\infty} e^{-\pi t y^{2} m^{2}} \sum_{n-v_{1} \in \mathbb{Z}}(m x+n) \mathrm{e}\left(n u_{2}\right) e^{-\pi t(m x+n)^{2}} t^{s+\frac{1}{2}} \frac{d t}{t},
\end{aligned}
$$

which can be written

$$
\begin{aligned}
A_{2}= & y^{s} \sum_{\substack{m-v_{2} \in \mathbb{Z} \\
m \neq 0}} \mathrm{e}\left(-m\left(u_{1}+x u_{2}\right)\right) \\
& \times \int_{0}^{\infty} e^{-\pi t y^{2} m^{2}} \sum_{n-v_{1}-m x \in \mathbb{Z}} n \mathrm{e}\left(n u_{2}\right) e^{-\pi t n^{2}} t^{s+\frac{1}{2}} \frac{d t}{t} .
\end{aligned}
$$

By Poisson summation for $t>0$ and $a, b \in \mathbb{R}$ it follows that

$$
\sum_{n-a \in \mathbb{Z}} n e^{-\pi t n^{2}} \mathrm{e}(b n)=-i t^{-\frac{3}{2}} \mathrm{e}(a b) \sum_{n+b \in \mathbb{Z}} n e^{-\frac{\pi}{t} n^{2}} \mathrm{e}(a n),
$$

so from (18) we get that

$$
\begin{aligned}
A_{2}= & -i y^{s} \mathrm{e}\left(v_{1} u_{2}\right) \sum_{\substack{m-v_{2} \in \mathbb{Z} \\
m \neq 0}} \mathrm{e}\left(-m u_{1}\right) \sum_{\substack{n+u_{2} \in \mathbb{Z} \\
n \neq 0}} n \mathrm{e}\left(\left(v_{1}+m x\right) n\right) \\
& \times \int_{0}^{\infty} e^{-\pi t y^{2} m^{2}-\frac{\pi}{t} n^{2}} t^{s-1} \frac{d t}{t} .
\end{aligned}
$$

Employing the formula [4, p.384 \#9]:

$$
\int_{0}^{\infty} e^{-a t-\frac{b}{t}} t^{s} \frac{d t}{t}=2\left(\frac{b}{a}\right)^{s / 2} K_{s}(2 \sqrt{a b}) \quad(a, b>0)
$$

we easily get the first equation of (17), in view of the definition (14).

Consider now $B$. From (16)

$$
B=i y^{s+1} \sum_{\substack{m-v_{2} \in \mathbb{Z} \\ m \neq 0}} m \mathrm{e}\left(-m u_{1}\right) \int_{0}^{\infty} e^{-\pi t y^{2} m^{2}} \sum_{n-v_{1} \in \mathbb{Z}} \mathrm{e}\left(n u_{2}\right) e^{-\pi t(m x+n)^{2}} t^{s+\frac{1}{2}} \frac{d t}{t}
$$


By Poisson summation again for $t>0$ and $a, b \in \mathbb{R}$ :

$$
\sum_{n-a \in \mathbb{Z}} e^{-\pi t n^{2}} \mathrm{e}(b n)=t^{-\frac{1}{2}} \mathrm{e}(a b) \sum_{n+b \in \mathbb{Z}} e^{-\frac{\pi}{t} n^{2}} \mathrm{e}(a n),
$$

so for the sum inside the integral we have

$$
\begin{aligned}
& \sum_{n-v_{1} \in \mathbb{Z}} \mathrm{e}\left(n u_{2}\right) e^{-\pi t(m x+n)^{2}} \\
= & \mathrm{e}\left(-m x u_{2}\right) \sum_{n-v_{1} \in \mathbb{Z}} \mathrm{e}\left((n+m x) u_{2}\right) e^{-\pi t(m x+n)^{2}} \\
= & \mathrm{e}\left(-m x u_{2}\right) \sum_{n-v_{1}-m x \in \mathbb{Z}} \mathrm{e}\left(n u_{2}\right) e^{-\pi n^{2}} \\
= & \left.t^{-\frac{1}{2}} \mathrm{e}\left(-m x u_{2}\right) \mathrm{e}\left(\left(v_{1}+m x\right)\right) u_{2}\right) \sum_{n+u_{2} \in \mathbb{Z}} \mathrm{e}\left(\left(v_{1}+m x\right) n\right) e^{-\frac{\pi}{t} n^{2}} \\
= & t^{-\frac{1}{2}} \mathrm{e}\left(u_{2} v_{1}\right) \sum_{n+u_{2} \in \mathbb{Z}} \mathrm{e}\left(\left(v_{1}+m x\right) n\right) e^{-\frac{\pi}{t} n^{2}} .
\end{aligned}
$$

Thus

$$
\begin{aligned}
B= & i \mathrm{e}\left(u_{2} v_{1}\right) y^{s+1} \sum_{\substack{n+u_{2} \in \mathbb{Z} \\
m-v_{2} \in \mathbb{Z} \\
m \neq 0}} m \mathrm{e}\left(-m u_{1}+n v_{1}\right) \mathrm{e}(m n x) \int_{0}^{\infty} e^{-\pi t y^{2} m^{2}-\frac{\pi}{t} n^{2}} t^{s} \frac{d t}{t} \\
= & i\left(u_{2}\right) y^{1-s} \pi^{-s} \Gamma(s) \sum_{\substack{m-v_{2} \in \mathbb{Z} \\
m \neq 0}} \mathrm{e}\left(-m u_{1}\right) m|m|^{-2 s} \\
& +2 i y e\left(u_{2} v_{1}\right) \sum_{\substack{n+u_{2} \in \mathbb{Z} \\
m-v_{2} \in \mathbb{Z} \\
m, n \neq 0}} e\left(-m u_{1}+n v_{1}\right) m\left|\frac{m}{n}\right|^{-s} K_{s}(2 \pi|m n| y) \mathrm{e}(m n x),
\end{aligned}
$$

by (19). After changing variables in the second sum, using the fact that $K_{s}=K_{-s}$ and referring to (12), it follows that

$$
\begin{aligned}
B= & i\left(u_{2}\right) y^{1-s} \psi\left(s-\frac{1}{2} ;-u_{1}, v_{2}\right) \\
& -2 i y e\left(u_{2} v_{1}\right) \sum_{\substack{m-u_{2} \in \mathbb{Z} \\
n+v_{2} \in \mathbb{Z} \\
m, n \neq 0}} e\left(-m v_{1}+n u_{1}\right) n\left|\frac{m}{n}\right|^{s} K_{-s}(2 \pi|m n| y) \mathrm{e}(m n x) .
\end{aligned}
$$

Thus by (13) we derive the second equation in (17) and complete the proof of Theorem 1.

Before turning to the derivation of Bloch's formula (1), we remark that the method used in the proof of Theorem 1 can clearly be extended to get the Fourier expansion of the Eisenstein series given for any nonnegative integer $k$ by

$$
y^{s} \sum_{\substack{m-v_{2} \in \mathbb{Z} \\ n-v_{1} \in \mathbb{Z}}}^{\prime} \frac{\mathrm{e}\left(n u_{2}-m u_{1}\right)(m \tau+n)^{k}}{|m \tau+n|^{2 s+k}},
$$

which still satisfies a nice functional equation $[7, \mathrm{p} .71]$. 


\section{Specialization}

In order to obtain Bloch's formula (1) from Theorem 1, we will show that

$$
\begin{aligned}
-i \pi y^{\frac{1}{2}} F_{u, 0}\left(\frac{3}{2}, \tau\right) & =J(q ; z) \quad \text { and } \\
\pi y^{\frac{1}{2}} F_{0, u}\left(-\frac{1}{2}, \tau\right) & =D(q ; z) .
\end{aligned}
$$

We may assume that $0 \leqslant u_{1}, u_{2}<1$. Applying (5) we get

$$
F_{u, 0}\left(\frac{3}{2}, \tau\right)=y^{\frac{3}{2}} \psi\left(\frac{3}{2} ; u_{2}, 0\right)-i y^{\frac{1}{2}} \sum_{\substack{m, n+u_{2} \in \mathbb{Z} \\ m, n \neq 0}} \mathrm{e}\left(-m u_{1}\right) \mathrm{e}(m n x) \frac{n}{|m|} e^{-2 \pi|m n| y},
$$

where $\psi\left(\frac{3}{2} ; u_{2}, 0\right)=\frac{2 i}{\pi^{2}} \sum_{n \geqslant 1} \frac{\sin 2 \pi u_{2} n}{n^{3}}=\frac{4 \pi i}{3} B_{3}\left(u_{2}\right)$. Thus

$$
\begin{aligned}
& -i \pi y^{\frac{1}{2}} F_{u, 0}\left(\frac{3}{2}, \tau\right) \\
= & \frac{4 \pi^{2} y^{2}}{3} B_{3}\left(u_{2}\right)-\sum_{n+u_{2} \in \mathbb{Z}}(-2 \pi n y) \log \left|1-\mathrm{e}\left(-u_{1}+n x+i|n| y\right)\right| \\
= & \frac{4 \pi^{2} y^{2}}{3} B_{3}\left(u_{2}\right)-\sum_{\ell \geqslant 1} \log \left|q^{\ell} z^{-1}\right| \log \left|1-q^{\ell} z^{-1}\right|+\sum_{\ell \geqslant 0} \log \left|q^{\ell} z\right| \log \left|1-q^{\ell} z\right|,
\end{aligned}
$$

after writing $n=\ell-u_{2}$ and splitting the sum over $n$ into $n>0$ and $n<0$. This proves (21) in view of (4) and the fact that for $0 \leqslant x<1$,

$$
B_{3}(x)=x^{3}-\frac{3}{2} x^{2}+\frac{1}{2} x
$$

Turning next to (22), we first employ [4, p.978]

$$
K_{\frac{3}{2}}(t)=\sqrt{\frac{\pi}{2 t}} e^{-t}\left(1+\frac{1}{t}\right)
$$

and obtain, after using (13) in (14), that

$$
\begin{aligned}
\pi y^{1 / 2} F_{0, u}\left(-\frac{1}{2}, \tau\right)= & \left(u_{2}\right) \pi i \psi\left(1 ;-u_{1}, 0\right) \\
& -\pi i y \sum_{\substack{m-u_{2}, n \in \mathbb{Z} \\
m, n \neq 0}} \mathrm{e}\left(n u_{1}\right) \mathrm{e}(m n x) e^{-2 \pi|m n| y}\left(1+\frac{1}{2 \pi|m n| y}\right) \frac{n|m|}{|n|^{2}} \\
= & -\frac{i}{2} \sum_{\substack{m-u_{2}, n \in \mathbb{Z} \\
n \neq 0}} \mathrm{e}\left(n u_{1}\right) \mathrm{e}(m n x) e^{-2 \pi|m n| y}\left(2 \pi y \frac{n|m|}{|n|^{2}}+\frac{n}{|n|^{3}}\right) \\
= & \sum_{m-u_{2} \in \mathbb{Z}}\left(-2 \pi|m| y \operatorname{Im} \log \left(1-\mathrm{e}\left(u_{1}+m x+i y|m|\right)\right)\right. \\
& \left.+\operatorname{Im}_{\operatorname{Li}}\left(\mathrm{e}\left(u_{1}+m x+i y|m|\right)\right)\right)
\end{aligned}
$$


where $\operatorname{Li}_{2}(x)=\sum_{n=1}^{\infty} \frac{x^{n}}{n^{2}}$, for $|x| \leqslant 1$. Hence by (3)

$$
\begin{aligned}
\pi y^{1 / 2} F_{0, u}\left(-\frac{1}{2}, \tau\right) & =\sum_{m-u_{2} \in \mathbb{Z}} D\left(\mathrm{e}\left(u_{1}+m x+i y|m|\right)\right) \\
& =\sum_{m-u_{2} \in \mathbb{Z}} D\left(\mathrm{e}\left(u_{1}+m x+i m y\right)\right)=\sum_{\ell \in \mathbb{Z}} D\left(q^{\ell} z\right)=D(q ; z),
\end{aligned}
$$

upon using the identity $D(\mathrm{e}(\bar{z}))=D(\mathrm{e}(z))$ and writing $\ell=m-u_{2}$. This proves (22) and hence Bloch's formula (1).

Finally, we remark that when $s=1 / 2$, the formula of Theorem 1 can be evaluated in terms of geometric series, after applying (5) again. In particular, this gives Hecke's [5] well-known formula for the Fourier expansion of a holomorphic Eisenstein series of weight 1 (see also [6]). Generally, when $s=1 / 2+\ell$ for $\ell \in \mathbb{Z}^{+}$, we can use Theorem 1 to evaluate $E_{u, v}(s, \tau)$ in terms of higher logarithms, at least when $v_{2}=0$. For this we use the following easily proved extension of (5) and (23) to any $\ell \geqslant 0$ :

$$
K_{\ell+\frac{1}{2}}(t)=\sqrt{\frac{\pi}{2 t}} e^{-t} \sum_{n=0}^{\ell} \frac{(\ell+n) !}{(\ell-n) ! n !}(2 t)^{-n} .
$$

Such a formula for $E_{u, 0}(s, \tau)$ is known from Theorem 1 of [8].

\section{References}

[1] B.C. Berndt, H. Chan, A. Zaharescu, A quasi-theta product in Ramanujan's lost notebook, Math. Proc. Cambridge Philos. Soc. 135 (2003), 11-18.

[2] S. Bloch, Higher Regulators, Algebraic K-theory, and Zeta Functions of Elliptic Curves, CRM Monograph series, 11, 2000.

[3] S. Chowla, A. Selberg, On Epstein's zeta-function J. Reine Angew. Math. 227 (1967), 86-110.

[4] I.S. Gradshteyn, I.M. Ryzhik, Tables of Integrals, Series and Products Fifth edition, Academic Press, London, 1994.

[5] E. Hecke: Theorie der Eisensteinschen Reihen höherer Stufe und ihre Anwendung auf Funkionentheorie und Arithmetik, Abh. Math. Sem. Hamb. 5 (1927), 199-224, in Math. Werke, Vandenhoeck \& Ruprecht, Göttingen, 461-486.

[6] E. Hecke: Bestimmung der Perioden gewisser Integrale durch die Theorie der Klassenkörper, Math. Zeit. 28 (1928), 708-727, in Math. Werke, Vandenhoeck \& Ruprecht, Göttingen, 505-524.

[7] C.L. Siegel: Lectures on advanced analytic number theory, Tata Institute, 1961. 
[8] D. Zagier, The Bloch-Wigner-Ramakrishnan Polylogarithm function, Math. Ann. 286 (1990), 613-624.

[9] A. Weil, Elliptic functions according to Eisenstein and Kronecker, Springer-Verlag, Berlin, 1976.

Addresses: W. Duke, UCLA Mathematics Dept., Box 951555, Los Angeles, CA 90095, USA Ö. Imamo ḡlu, ETH, Mathematics Dept. CH-8092, Zürich, Switzerland

E-mail: wdduke@ucla.edu; ozlem@math.ethz.ch

Received: 11 July 2006; revised: 10 August 2006 